\title{
Label-free quantitative proteomics and bioinformatics analyses of alcoholic liver disease in a chronic and binge mouse model
}

\author{
YU ZHANG $^{1 *}, \mathrm{CHENG} \mathrm{ZHAN}^{2 *}$, GENWEN CHEN $^{1}$ and JIANYONG SUN ${ }^{1}$ \\ Departments of ${ }^{1}$ Gastroenterology and ${ }^{2}$ Thoracic Surgery, Zhongshan Hospital, Fudan University, Shanghai 200032, P.R. China
}

Received September 20, 2017; Accepted February 14, 2018

DOI: $10.3892 / \mathrm{mmr} .2018 .9225$

\begin{abstract}
As a significant cause of mortality and morbidity, alcoholic liver disease (ALD) has been widely investigated. However, little is known about the underlying metabolic mechanisms involved in the complicated pathological processes of ALD. The present study used label-free quantitative proteomics and bioinformatics analyses to investigate the differentially expressed proteins (DEPs) and their functions in the livers of alcohol-feed (AF) and control pair-feed (PF) mice. As a result, 87 upregulated DEPs and 133 downregulated DEPs were identified in AF liver tissues compared with PF livers. Gene ontology and Kyoto encyclopedia of genes and genomes bioinformatics analyses demonstrated that the DEPs were significantly enriched in 'protein binding', 'metabolism', 'signal conduction' and 'immune response'. The expression of several core proteins including thyroid hormone receptor interactor 12 (TRIP12), NADH dehydrogenase (ubiquinone)1 $\alpha$ subcomplex, assembly factor 3 (NDUFAF3) and guanine monophosphate synthetase (GMPS) was validated by reverse transcription-quantitative polymerase chain reaction (RT-qPCR) in a larger series of samples. The RT-qPCR results confirmed that TRIP12, NDUFAF3 and GMPS genes were significantly differentially expressed in between the $\mathrm{AF}$ and PF samples. These results extend our understanding of the molecular mechanisms underlying the occurrence and development of ALD. The present study indicated that the majority of DEPs serve vital roles in multiple metabolic pathways and this extends our knowledge of the molecular mechanisms involved in the occurrence and progression of ALD.
\end{abstract}

Correspondence to: Dr Jianyong Sun, Department of Gastroenterology, Zhongshan Hospital, Fudan University, 180 Fenglin Road, Xu Hui, Shanghai 200032, P.R. China

E-mail: sjylaoshiketizu@163.com

${ }^{*}$ Contributed equally

Key words: alcoholic liver disease, alcohol-feed mice, control pair-feed mice, differentially expressed proteins, gene ontology analysis, kyoto encyclopedia of genes and genomes analysis

\section{Introduction}

Alcoholic liver disease (ALD) has been one of the leading causes of cirrhosis and liver-related mortality and morbidity worldwide for many years $(1,2)$. As the ultimate outcome of heavy acute and/or chronic alcohol drinking, ALD can lead to steatosis, steatohepatitis, alcoholic fibrosis, cirrhosis and even hepatocellular carcinoma in certain individuals $(3,4)$. Many factors are thought to contribute to the development and progression of ALD, particularly the toxicity of alcohol and its metabolites, generation of reactive oxygen species during alcohol metabolism, and endotoxin derived from the gut $(5,6)$. Although the factors that link ethanol to the occurrence and development of liver injury have been widely investigated, the underlying metabolic mechanisms involved in the complicated pathological processes remain to be elucidated. Thus, comprehensive research into the molecular characteristics and mechanism of ALD is urgently required, to identify effective treatment methods and improve the outcome of patients with ALD.

Label-free quantitative proteomics is a method that aims to determine the relative amount of proteins, which is a novel tool used for biomarker identification in various diseases, due to the critical importance of protein-level measurements (7-9). In the present study, label-free quantitative proteomics was used to detect ALD in a rodent model, to explore the underlying pathophysiological mechanism. It is hoped that the results of the present study can improve knowledge of the molecular pathogenesis of ALD and aid the search for biomarkers for early diagnosis and treatment.

\section{Materials and methods}

Animal model. All procedures of animal care and treatment were approved by the Institutional Animal Care and Use Committee of Fudan University (Shanghai, China). Experiments were performed on male specific-pathogen-free C57BL/6J mice (Laboratory Animal Center, Fudan University, Shanghai, China) of 6 weeks old weighing 18-20 g. Environmental conditions were strictly controlled (temperature $23 \pm 2^{\circ} \mathrm{C}$, relative humidity $50-70 \%$ and $12 \mathrm{~h} \mathrm{light/dark}$ cycle) with ad libitum access to food and water. Following 1 week acclimation, all mice were randomly divided into two groups based on diets as follows; the alcohol-feed (AF) and control pair-feed (PF) groups. 
Mice were administered 4\% Lieber-Decarli ethanol liquid diet (TP 4030B; Trophic Animal Feed High-Tech Co., Ltd. Nantong, China) and control diet (TP 4030C; Trophic Animal Feed High-Tech Co., Ltd.) respectively for 4 weeks with diets changed daily at $5 \mathrm{pm}(10)$. On the 29th day at 9:00 am, mice were administered by gavage with a single dose of maltose dextrin (control, $9 \mathrm{~g}$ maltose dextrin per $\mathrm{kg}$ of body weight) or ethanol diet (5 $\mathrm{g}$ ethanol diet per $\mathrm{kg}$ of body weight), respectively. At $9 \mathrm{~h}$ after the binge, the mice were anesthetized by an intraperitoneal injection of $1 \%$ pentobarbital sodium $(80 \mathrm{mg} / \mathrm{kg}$ body weight) and the serum and liver were collected (11). There were 10 mice in each group. All the non-alcoholic and alcoholic diets were provided throughout the sample collection period following the binge. A dim red light was used to collect tissues in dark conditions.

Histopathological examination. The liver tissues were resected and divided to three parts and processed as follows: Paraffin embedded and stained with hematoxylin/eosin (H\&E); frozen sections $(10 \mu \mathrm{m})$ and stained with Oil Red O for $10-15 \mathrm{~min}$ at $37^{\circ} \mathrm{C}$; and immediately frozen in liquid nitrogen and stored at $-80^{\circ} \mathrm{C}$ for further analysis. All sections were analyzed by light microscopy by at least two independent researchers.

Liquid chromatography-mass spectrometry/mass spectrometry (LC-MS/MS) analysis. There were three samples in every group and every sample $(5 \mathrm{ul})$ was mixed from three mouse liver tissues. Each sample was resuspended in buffer A ( $0.1 \%$ formic acid; FA). Separations were performed with an UltiMate 3000 HPLC system (Thermo Fisher Scientific, Inc., Waltham, MA, USA) and Q-Extractive HF HF-X Hybrid Quadrupole-Orbitrap Mass Spectrometer (Thermo Fisher Scientific, Inc.). The peptides were subjected to a $\mathrm{C} 18$ trap column $(3 \mu \mathrm{m}, 0.1 \times 20 \mathrm{~mm})$ at a flow rate of $0.6 \mu \mathrm{l} / \mathrm{min}$. Peptides were desalted online and loaded onto a C18 column $(1.9,150 \times 120 \mathrm{~mm})$ using a gradient from $6-95 \%$ buffer B (0.08\% FA and $80 \%$ acetonitrile) for $90 \mathrm{~min}$. The mass spectrometer was operated in positive mode using a data-dependent acquisition method.

A full MS scan (300-1,400 m/z) was acquired in the mass spectrometer with the resolution set to a value of 120,000 .

Identification of differently expressed proteins (DEPS). DEPs were identified according to the following procedures. Only the $\mathrm{FC}$ value $>1.5$ or $<0.667$ were entered in the following analyses. Then a random variance model $t$ test was performed using SPSS version 20.0 (IBM Corp., Armonk, NY, USA) to filter the DEPs as it can effectively increase the statistical effects in a small number of samples. Only proteins with $\mathrm{P}<0.05$ and false discovery rate (FDR) $<0.05$ were considered to be significantly differentially expressed, as previously reported $(12,13)$. A clustering analysis map was built using Cluster 3.0 software version 2.3 (Bio-Fly Bioscience; www. bangfeibio.com/company) to identify DEPs efficiently with similar expression mode.

Reverse transcription-quantitative polymerase chain reaction ( $R T-q P C R)$. The relative mRNA level in the liver tissue $(20 \mathrm{mg})$, including thyroid hormone receptor interactor 12 (TRIP12), NADH dehydrogenase (ubiquinone) $1 \alpha$ subcomplex, assembly factor 3 (NDUFAF3) and guanine monophosphate synthetase

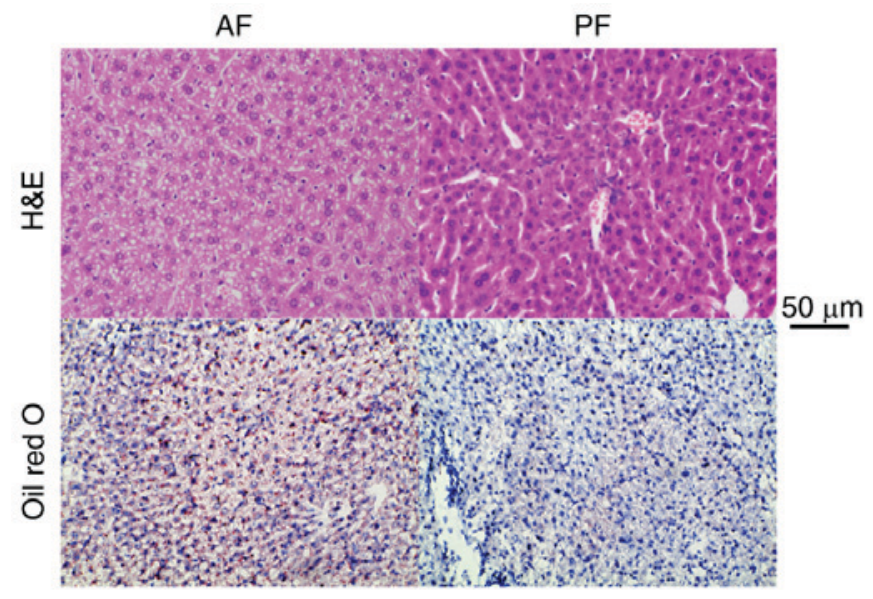

Figure 1. Effect of alcohol on liver histopathological alterations. Liver sections were stained with H\&E and Oil Red O to visualize lipid droplets (in red). Representative images (magnification, x200) of each group are presented. AF, alcohol-feed; PF, control pair-feed; H\&E, hematoxylin/eosin.

(GMPS), in the AF and PF groups were measured by RT-qPCR. Briefly, the total RNA was isolated using TRIzol reagent (Thermo Fisher Scientific, Inc.) according to the manufacturer's protocol and then RT was performed using GeneAmp RNA PCR kits (Thermo Fisher Scientific, Inc.) with the following parameters: $15 \mathrm{~min}$ at $37^{\circ} \mathrm{C}, 5 \mathrm{sec}$ at $85^{\circ} \mathrm{C}$, hold at $4^{\circ} \mathrm{C}$. Primer sequences are listed in Table I. qPCR was performed on an ABI 7500 real-time PCR thermocycler using SYBR-Green PCR Master Mix kits (Thermo Fisher Scientific, Inc.) with the following PCR cycling parameters: 1 cycle of $30 \mathrm{sec}$ at $95^{\circ} \mathrm{C}$; 40 cycles of $5 \mathrm{sec}$ at $95^{\circ} \mathrm{C} ; 30 \mathrm{sec}$ at $55^{\circ} \mathrm{C}$; and, $30 \mathrm{sec}$ at $72^{\circ} \mathrm{C}$ followed by a melting curve analysis. The expression levels of target genes were calculated from duplicate samples following normalization against the housekeeping gene GAPDH. The $2^{-\Delta \Delta \mathrm{Cq}}$ method was used to calculate the expression of these proteins (14).

Gene ontology (GO) and Kyoto Encyclopedia of Genes and Genomes (KEGG) pathway analyses. GO and KEGG analyses were performed to investigate significantly enriched function and signaling pathways of DEPs based on the GO (geneontology.org/) and KEGG databases (genome.jp/kegg/) as previously published $(15,16)$. Specifically, the GO and KEGG categories were classified using Fisher's exact test and $\chi^{2}$ test and FDR calculated for multiple testing corrections. Only GOs and signaling pathways with $\mathrm{P}<0.05$ and FDR $<0.05$ were considered statistically significant. A GO map and a path-net were established to further summarize the fundamental functional links among the significant GOs and KEGG pathways, respectively.

Cluster of orthologous groups of proteins analysis. The DEPs were compared based on the clusters of orthologous groups (COGs) database (ncbi.nlm.nih.gov/COG/), to categorize the orthologous and paralogs of protein. COGs category assignment was performed using the local alignment tool BLASTP with an e-value cutoff of $<104$. Only the categories that met a threshold of $\mathrm{P}<0.05$ and $\mathrm{FDR}<0.05$ were considered statistically significant. 
Table I. Primer sequences for the targeted proteins.

\begin{tabular}{lll}
\hline Gene name & \multicolumn{1}{c}{ Forward $\left(5^{\prime}>3 '\right)$} & Reverse $\left(5^{\prime}>3^{\prime}\right)$ \\
\hline TRIP12 & GTCTGTGACGCAGGACCTTG & TGTGAACTGGCTTAGCTGTCCT \\
NDUFAF3 & GTGGTCCAGTGGAACGTGG & CTCCTGTCACTCGACCTTCG \\
KDSR & GCTCCTCTACATGGTGTCGC & CTCAATAGCAATGCACTTCCCA \\
SURF6 & CTGAACGACAGAGGAGCACAT & TTGGGCCTAGAAGAGGTAGGA \\
KIF5B & GCGGAGTGCAACATCAAAGTG & CATAAGGCTTGGACGCGATCA \\
GMPS & GATGCAGTGGGAACTTTACTGT & AGCACGATTTAGCAAAGCTGT \\
DDAH2 & GCAGGTAGTAGAACGGAAGATCC & CTGGTGACAATGGAAGGCTCA \\
SSRP1 & CAGAGACATTGGAGTTCAACGA & GCCCGTCTTGCTGTTCTTAAAG \\
HIST1HIC & AACCCCAGGCTAAGAAGGC & TGGCTTTACGGCTTTAGACGC \\
H1F0 & CACGGACCACCCCAAGTATTC & ACCCACCTTGTAGTGGCTCT
\end{tabular}

TRIP12, thyroid hormone receptor interactor 12; NDUFAF3, NADH dehydrogenase (ubiquinone) $1 \alpha$ subcomplex, assembly factor 3; KDSR, 3-ketodihydrosphingosine reductase; SURF6, surfeit gene 6; KIF5B, kinesin family member 5B; GMPS, guanine monophosphate synthetase; DDAH2, dimethylarginine dimethylaminohydrolase; SSRP1, structure specific recognition protein 1; HIST1HIC, histone cluster 1 H1c; H1F0, H1 histone family member 0 .
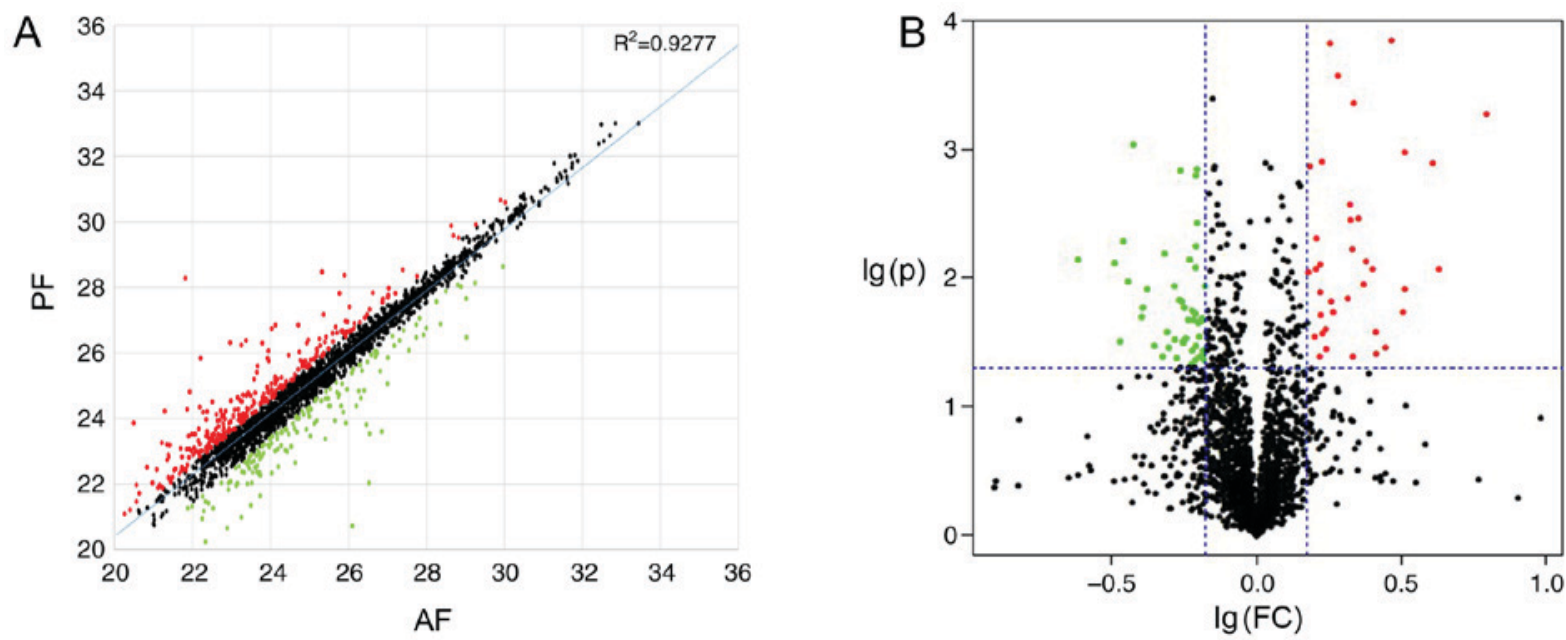

Figure 2. Identification of DEPs between AF and PF groups. (A) The scatter plot for global protein expression between the two groups; red dots represent correlation coefficients ( $>1.5$ times) of $\mathrm{AF} / \mathrm{PF}$ and green dots represent that of $\mathrm{PF} / \mathrm{AF}$. (B) Volcano plots represented all the genes in $\mathrm{AF}$ and $\mathrm{PF}$ groups according to P-value and fold changes; black dots represent genes that were not differentially expressed, while red dots and green dots represent DEPs. DEPs, differentially expressed proteins; AF, alcohol-feed mice; PF, control pair-feed mice.

\section{Results}

Hepatic steatosis and histopathological examination. Compared with PF group, the AF mice exhibited marked hepatic steatosis, even steatohepatitis, as evaluated by $\mathrm{H} \& \mathrm{E}$ and Oil Red O (Fig. 1). Histopathological examination revealed the accumulation of lipid vacuoles and lipid droplets in the AF mice. This demonstrated the animal model of the present study was successful.

DEPs between the AF and PF mice. A total of 87 upregulated DEPs and 133 downregulated DEPs were identified in the AF group compared with the PF group. A scatter plot map and a volcano plot were established to demonstrate all differentially expressed proteins (Fig. 2). As Fig. 3 presents, marked differences ( $>40$-fold changes) were detected in the expression levels of certain proteins, which may have potential to be used as biomarkers in the diagnosis, assessment and monitoring of ALD. Fig. 4 indicated that the relative mRNA level of TRIP12, NDUFAF3 and GMPS were significantly different in AF and PF. The expression of all 220 DEPs were listed in a clustering map, to identify proteins with similar expression modes between AF and PF groups (Fig. 5).

GO analysis. The significantly enriched GO terms $(\mathrm{P}<0.01$ only) are summarized in Table II. The significant GOs were categorized according to molecular function, biological processes and cellular component. When the molecular function of these DEPs was analyzed, the majority of DEPs were associated with binding functions, including protein binding, ion binding and nucleic acid binding. In the biological processes analysis of GO, the majority of DEPs were associated with 'metabolic process', including the 'macromolecule metabolic process', 'primary metabolic 


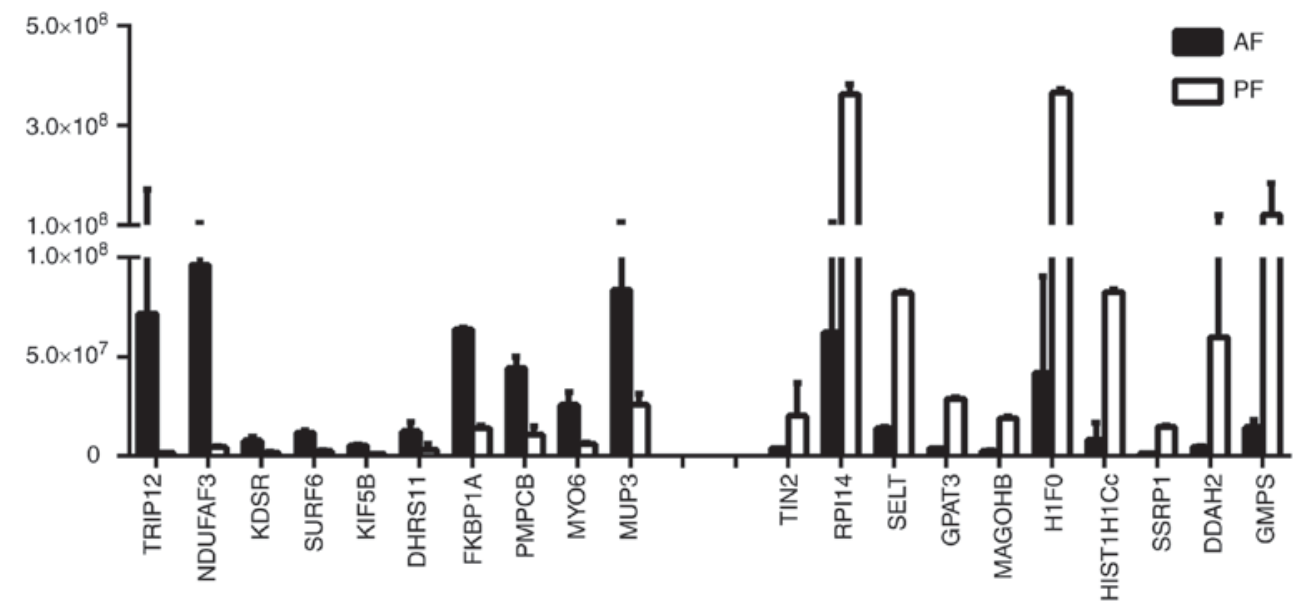

Figure 3. The 10 proteins most strongly upregulated and downregulated in alcoholic liver disease. The ordinate value represents the relative abundance of the proteins $(\mathrm{P}<0.05$, false discovery rate $<0.05)$. AF, alcohol-feed mice; $\mathrm{PF}$, control pair-feed mice.

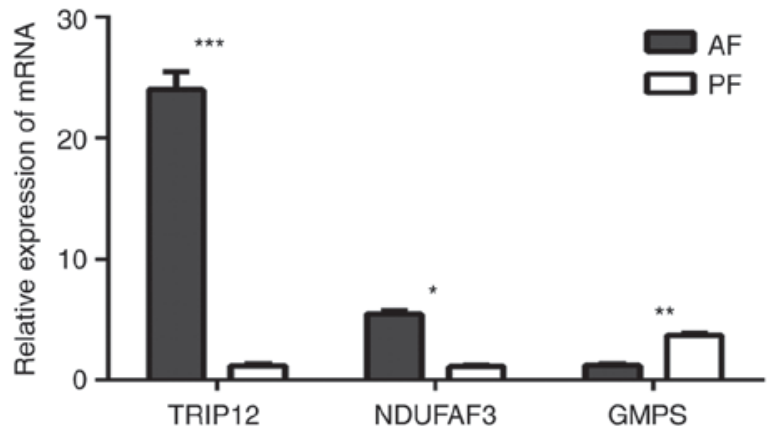

Figure 4. Relative expression of the mRNA level of the selected proteins. ${ }^{*} \mathrm{P}<0.05,{ }^{* *} \mathrm{P}<0.01$ and ${ }^{* * *} \mathrm{P}<0.001, \mathrm{AF}$ vs. PF. AF, alcohol-feed mice; $\mathrm{PF}$, control pair-feed mice; TRIP12, thyroid hormone receptor interactor 12; NDUFAF3, NADH dehydrogenase (ubiquinone) $1 \alpha$ subcomplex, assembly factor 3; GMPS, guanine monophosphate synthetase.

process' and 'cellular metabolic process'. In addition, when the DEPs were analyzed for cellular components, they were enriched in mitochondria and ribosomes (data not shown). A hierarchical tree of the GO terms was established according to their associations (Figs. 6 and 7).

KEGG pathway analysis. As demonstrated in Fig. 8, the KEGG analysis results demonstrated that the DEPs were significantly enriched in 'adenosine monophosphate activated protein kinase (AMPK) signaling pathway', 'Ras signaling pathway', 'Notch signaling pathway', 'p53 signaling pathway' and 'autophagy' (Fig. 8). These results indicated that ALD was associated with several biological processes, such as dysregulated lipid and glucose metabolism, catabolic processing, cell-cell adhesion, cell amplification. Additionally, these data indicated that the intervention of these pathways may provide ways for molecular targeting therapies of ALD.

COGs analysis. According to the COGs analysis, the functions of the DEPs were enriched in 'information storage and processing', 'cellular processes and signaling' and 'metabolism' (Fig. 9). The results indicated that these processes, particularly the metabolism of lipids, amino acids and nucleic acid, served important roles in the development of ALD.

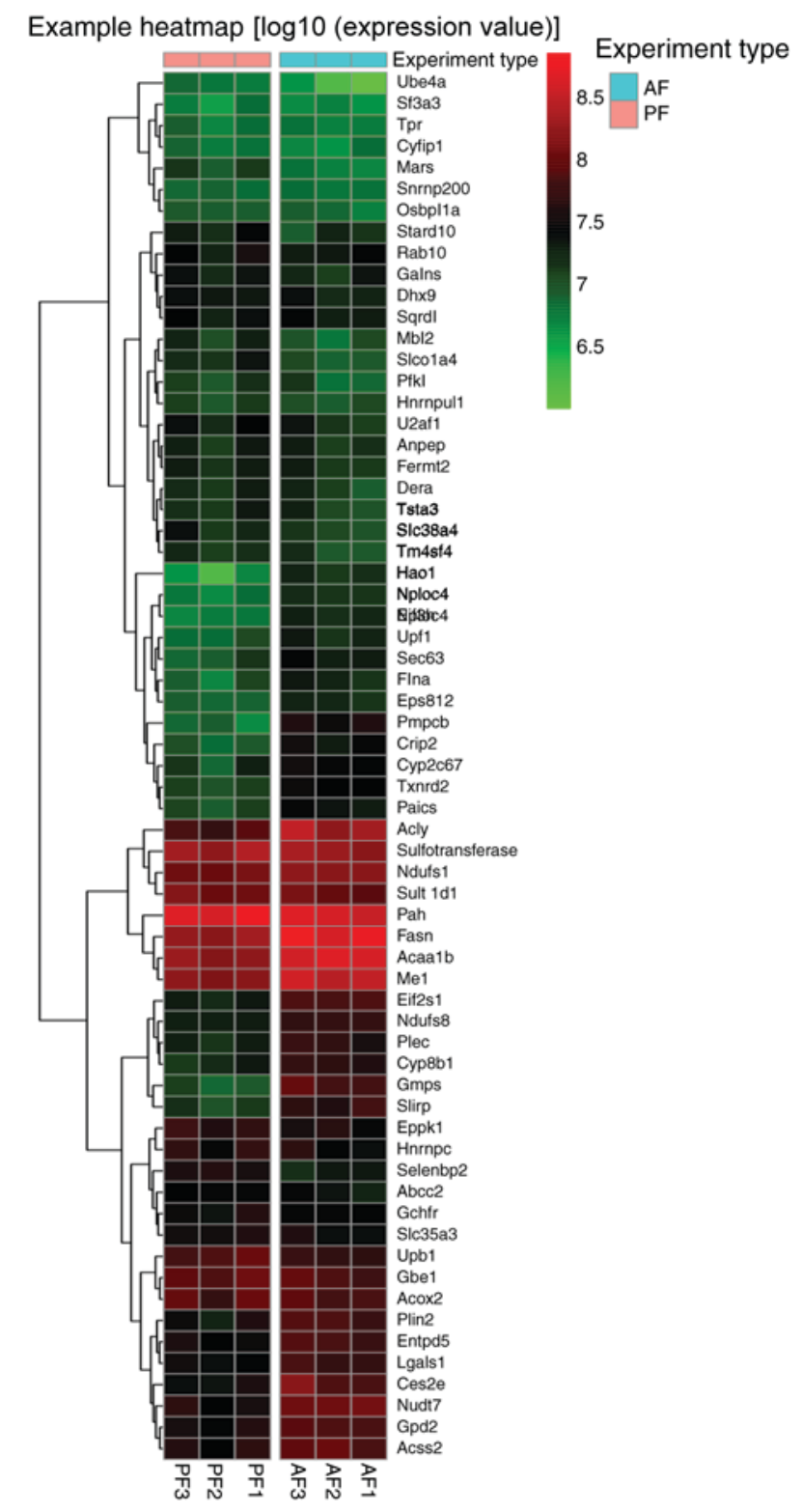

Figure 5. Clustering analysis of DEPs between the two groups. Red represents upregulation of DEPs and green represents downregulation of DEPs. DEPs, differentially expressed proteins; AF, alcohol-feed mice; PF, control pair-feed mice. 
Table II. Enriched GO terms in biological process $(\mathrm{P}<0.01)$.

\begin{tabular}{|c|c|c|c|c|}
\hline GO ID & Description & Input number & Input all & P-value \\
\hline GO:0010467 & Gene expression & 127 & 718 & 0.001414742 \\
\hline GO:0090304 & Nucleic acid metabolic process & 96 & 718 & 0.000287556 \\
\hline GO:0016070 & RNA metabolic process & 85 & 718 & 0.000248985 \\
\hline GO:0003677 & DNA binding & 46 & 718 & 0.002166795 \\
\hline GO:0007005 & Mitochondrion organization & 46 & 718 & 0.002575995 \\
\hline GO:0006351 & Transcription, DNA-templated & 37 & 718 & 0.001753421 \\
\hline GO:0032774 & RNA biosynthetic process & 37 & 718 & 0.001753421 \\
\hline GO:0097659 & Nucleic acid-templated transcription & 37 & 718 & 0.001753421 \\
\hline GO:0000313 & Organellar ribosome & 22 & 718 & $7.62 \mathrm{E}-08$ \\
\hline GO:0005761 & Mitochondrial ribosome & 22 & 718 & $7.62 \mathrm{E}-08$ \\
\hline GO:0045321 & Leukocyte activation & 16 & 718 & 0.001056161 \\
\hline GO:0044801 & Single-organism membrane fusion & 11 & 718 & 0.001415192 \\
\hline GO:0048284 & Organelle fusion & 11 & 718 & 0.002478183 \\
\hline GO:0000315 & Organellar large ribosomal subunit & 10 & 718 & 0.000209737 \\
\hline GO:0005762 & Mitochondrial large ribosomal subunit & 10 & 718 & 0.000209737 \\
\hline GO:0000149 & SNARE binding & 9 & 718 & 0.000643314 \\
\hline GO:0005085 & Guanyl-nucleotide exchange factor activity & 9 & 718 & 0.000643314 \\
\hline GO:0000314 & Organellar small ribosomal subunit & 9 & 718 & 0.001423751 \\
\hline GO:0005763 & Mitochondrial small ribosomal subunit & 9 & 718 & 0.001423751 \\
\hline GO:0090174 & Organelle membrane fusion & 8 & 718 & 0.000805555 \\
\hline GO:0031201 & SNARE complex & 8 & 718 & 0.000805555 \\
\hline GO:0005484 & SNAP receptor activity & 7 & 718 & 0.000265346 \\
\hline GO:0006906 & Vesicle fusion & 7 & 718 & 0.002514721 \\
\hline GO:0005088 & Ras guanyl-nucleotide exchange factor activity & 6 & 718 & 0.000988442 \\
\hline
\end{tabular}

GO, Gene Ontology; SNARE, SNAP receptor; SNAP, soluble NSF attachment protein.

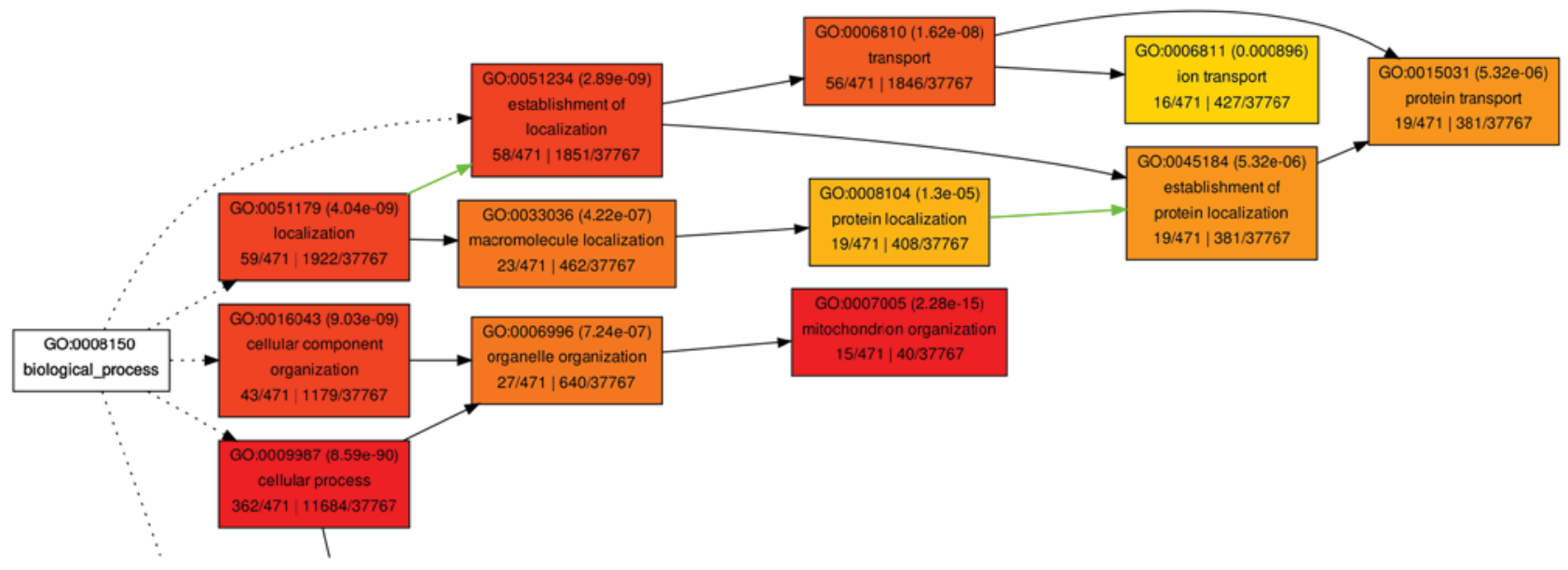

Figure 6. First part of the GO map in alcoholic liver disease. GO analysis results $(\mathrm{P}<0.01$, false discovery rate $<0.05)$. Deeper color of the GO terms represents greater significance in regulating the downstream GOs. GO, Gene Ontology.

\section{Discussion}

High-throughput quantitative proteomics has emerged as a popular method in the search for disease-associated factors using high-throughput analysis in recent years (12,17-19). However, to the best of the authors' knowledge, the present study is the first to use high-throughput quantitative proteomics analysis in a mouse model of ALD to identify the core proteins directly, rather than mRNAs. The chronic-plus-binge alcohol feeding model in mice, which mimics the drinking pattern of patients with alcoholic hepatitis with a background of drinking for a number of years (chronic) and a history of recent excessive alcohol consumption (binge), is now frequently used worldwide (20-23). In the present study, the ALD model in 


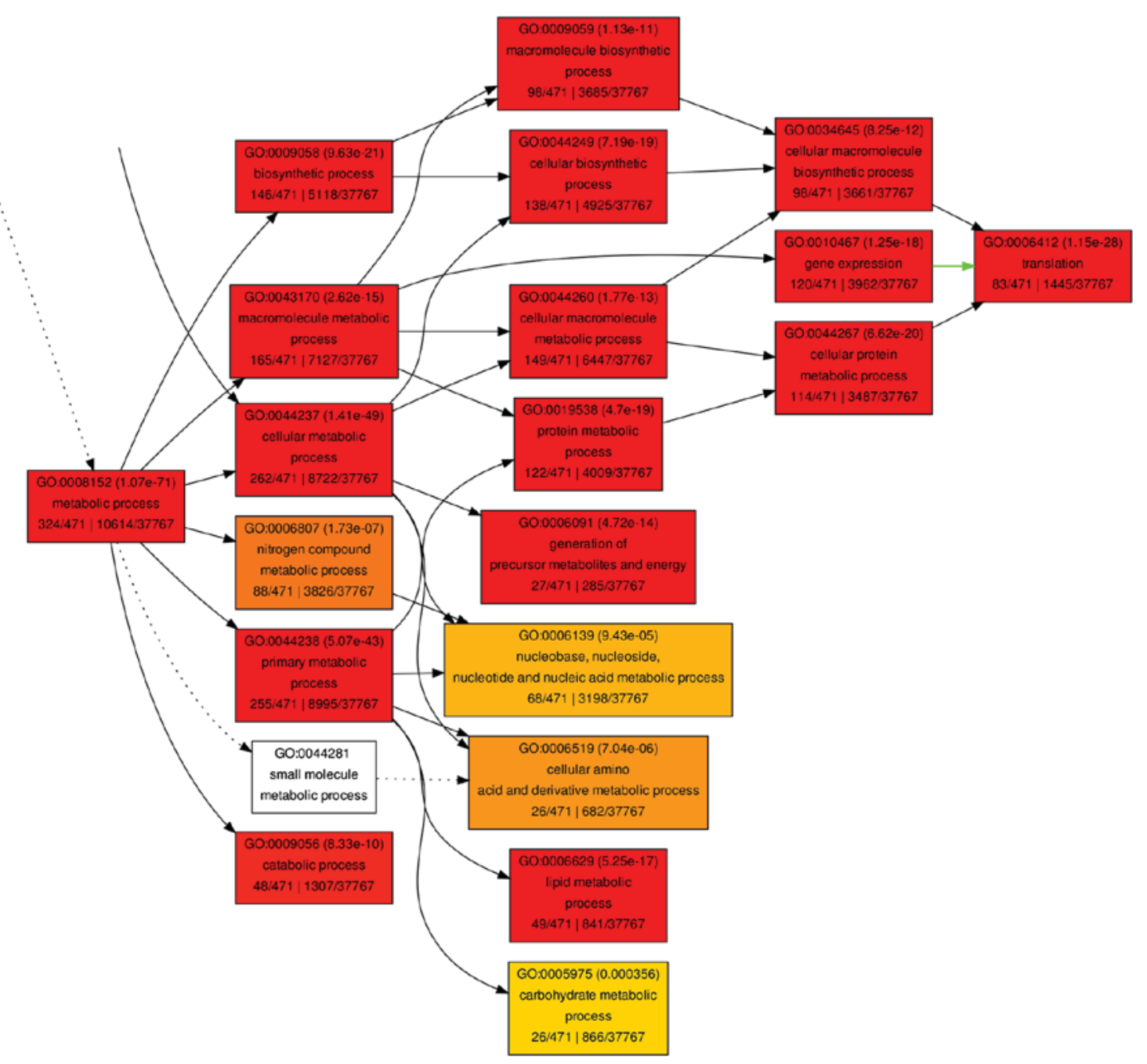

Figure 7. Second part of the GO map in alcoholic liver disease. GO, Gene Ontology.

mice with the liver steatosis and inflammation was successfully established using this method.

Label-free quantitative proteomics quantifies peptides and proteins without the use of stable-isotope labels. It directly uses a peptide's response (intensity) in the mass spectrometer as a quantitative measure and infers quantity indirectly from the number of peptide-to-spectrum matches obtained for each protein $(24,25)$. The present study identified 220 DEGs in the ALD mice. These DEPs may be used as characteristic proteins in the diagnosis and treatment of ALD. Several of these DEPs have been previously described in ALD progression. For example, acyl-CoA dehydrogenase is involved in mitochondrial b-oxidation of fatty acids in the ALD progress $(26,27)$. Glutathione-S-trans-ferases, which are responsible for the detoxification of potentially toxic by-products of ethanol metabolism, including acetaldehyde and reactive oxygen species (ROS), were also identified as downregulated in the AF mice (28). Eaton et al (29) reported that chronic alcohol ingestion leads to downregulation of NADH dehydrogenase (ubiquinone), consistent with the findings of the present study. Decreased amounts of ubiquinone can lead to fat accumulation and increase in free radical damage, leading to liver injury $(30,31)$. In the present study, ATP-citrate synthase was upregulated, which catalyzes the exchange of ADP and

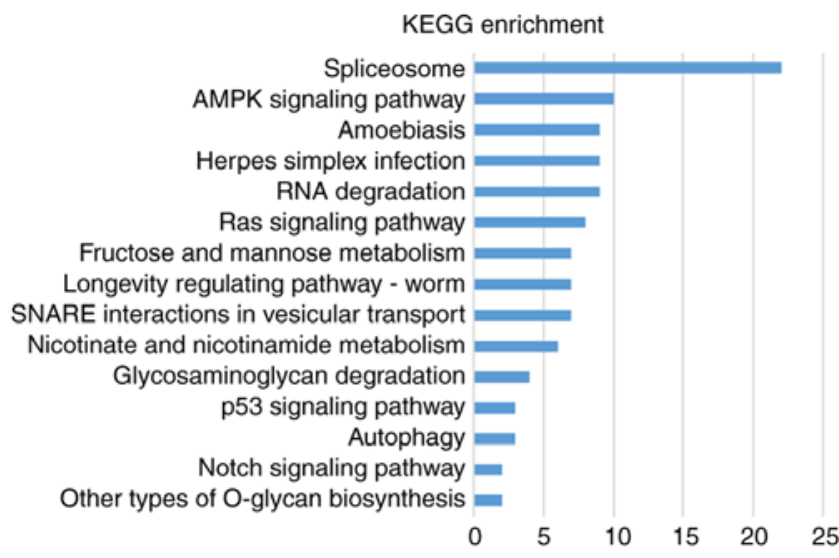

Figure 8. Pathway enrichment analysis. The enrichment bar chart of significant pathways is shown. As the enrichment increases, the corresponding function is more specific. KEGG, Kyoto Encyclopedia of Genes and Genomes; AMPK, adenosine monophosphate activated protein kinase; SNARE, soluble NSF attachment protein receptor.

ATP across the mitochondrial membrane and supports the increase in ATP generation during ALD (32). The ubiquitin system has been mechanistically implicated in a number of human diseases including cancers and ALD (33). The 


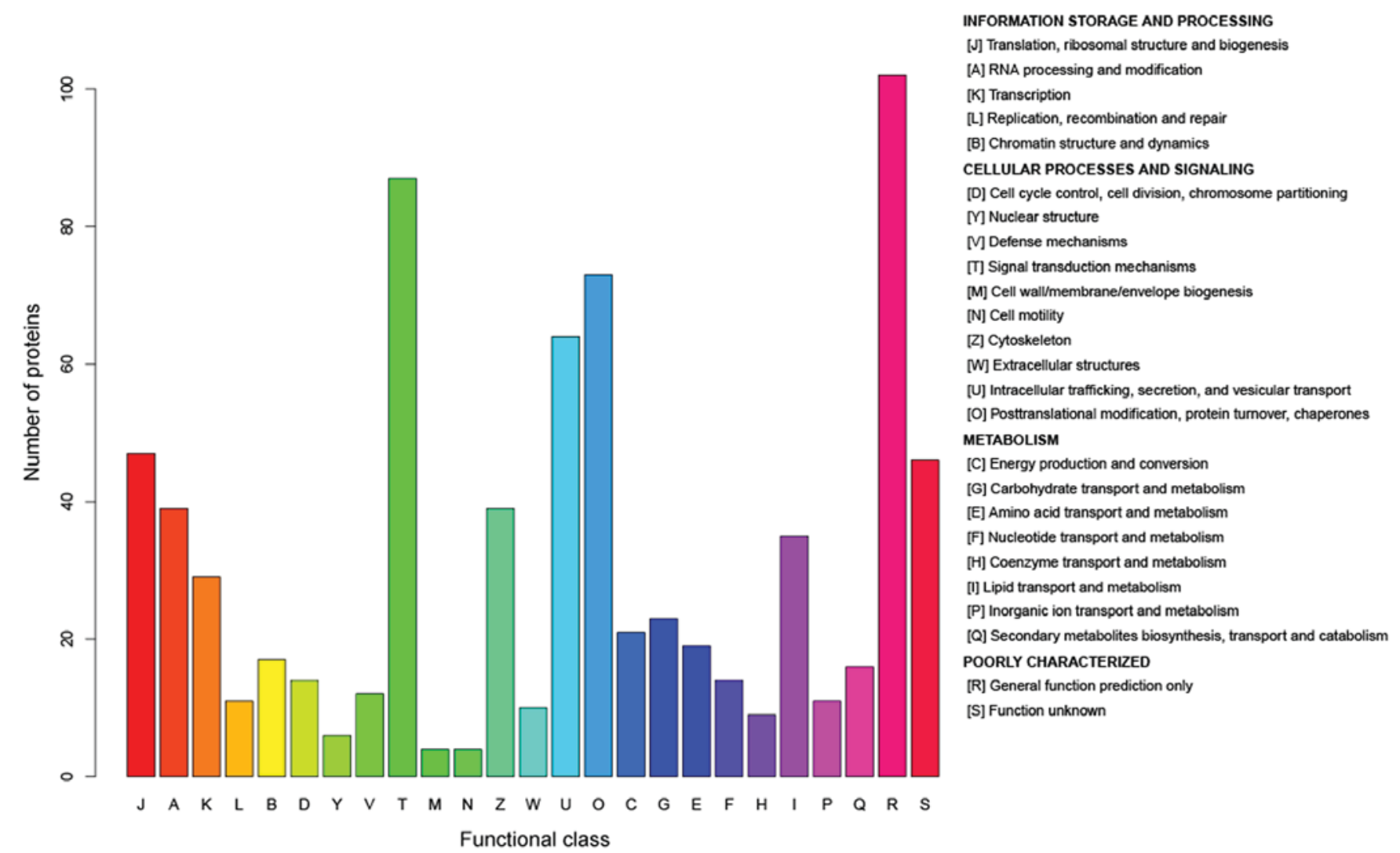

Figure 9. Clusters of orthologous groups analysis of differentially expressed proteins, the different colors represent different functions. The value of the ordinate represents the number of proteins enriched in this function.

ubiquitin system protein E3 ubiquitin-protein ligases TRIP12, deltex 3-like E3 ubiquitin ligase, HECT domain E3 ubiquitin protein ligase 3 (HECTD3), HECTD1 and ring finger protein 114, which were identified as differently expressed in the present study, were associated with cellular response to DNA damage stimulus, a ubiquitin-dependent protein catabolic process. This result suggested that the ubiquitin system is worthy of more attention in the exploration of ALD. The ethanol-inducible P450, a member of the cytochrome P450 multifamily, is a major component of the microsomal ethanol oxidizing system, which metabolizes a small portion of ethanol $(34,35)$. Previous studies have reported that hepatic expression of the cytochrome P450 family 2 subfamily E member 1 (CYP2E1) mRNA and/or expression of the CYP2E1 protein are increased in different physiological or pathological conditions, including fasting, a high fat diet, diabetes, obesity or ethanol intoxication $(36,37)$. Accumulation of ROS due to increased hepatic CYP2E1 expression may lead to lipid peroxidation of cellular membranes; antioxidant depletion also causes oxidative stress then damage liver DNA and contributes to hepatic fibrosis (38-40). The current study identified that cytochrome P450, family 2, subfamily c, polypeptide 67 and CYP2E1 were all upregulated in the AF mice.

Using GO and KEGG analyses, the present study identified various gene functions and signaling pathways that were significantly altered in ALD, including lipid metabolism, inorganic ion metabolism, and the AMPK and p53 signaling pathways. These GOs and pathways may serve critical roles in ALD. Notably, it was identified that a number of GO terms were associated with protein and ion metabolism, including 'iron transport', 'protein transport' and 'protein localization'. Particularly, 13 of the DEPs were identified to be associated with the 'iron ion binding', including the upregulated DEP, transferrin receptor 1, and downregulated DEP, hepcidin, which has been reported to induce the overload of iron in ALD in previous studies (41-43). Additionally, it was identified that transferrin receptor protein 2 was increased and hepcidin-2 decreased in AF mice compared with PF mice. Increasing evidence indicates that AMPK regulates sterol regulatory element binding transcription factor 1, which is involved in the control of glucose, lipid and cholesterol metabolism and participates in the pathogenesis of hepatic steatosis (44). The DEPs involved in the AMPK signaling pathway, including fatty acid synthase, ATP-dependent 6-phosphofructokinase, Acyl-CoA desaturase and phosphatidylinositol 3-kinase regulatory subunit $\alpha$, may be the core proteins in the progression of ALD.

The system of COGs was designed to accommodate the extremely different evolution rates observed for different genes and to comprise a framework for functional and evolutionary genome analysis (45). According to the COGs analysis, the functions of the DEPs were predominantly enriched in 'information storage and processing', 'cellular processes and signaling', in addition to 'metabolism' including 'lipid transport and metabolism' and 'inorganic ion transport and metabolism'. The results COGs supported GO and pathway analyses, revealing that proteins associated with metabolism had an important role in ALD. 
In conclusion, label-free quantitative proteomics using LC-MS/MS was performed to identify DEPs between AF and control PF mice livers. The present study suggested that certain DEPs were involved in the response to alcohol and that the core proteins identified the present study may be useful to predict the development of ALD. It is hoped that the findings will be further validated in other experiments in the near future.

\section{Acknowledgements}

Not applicable.

\section{Funding}

The present study was funded by the Science and Technology Commission Foundation of Shanghai (grant no. 13DZ1930908).

\section{Availability of data and materials}

The datasets used or analyzed during the current study are available from the corresponding author on reasonable request.

\section{Authors' contributions}

JS and YZ conceived and designed the study. $\mathrm{YZ}, \mathrm{CZ}$ and $\mathrm{GC}$ performed the experiments. $\mathrm{YZ}$ and $\mathrm{CZ}$ wrote the paper. JS, YZ, CZ and GC reviewed and edited the manuscript. All authors read and approved the manuscript.

\section{Ethics approval and consent to participate}

All procedures of animal care and treatment were approved by the Institutional Animal Care and Use Committee of Fudan University (Shanghai, China).

\section{Patient consent for publication}

Not applicable.

\section{Competing interests}

The authors declare that they have no competing interests.

\section{References}

1. Rehm J, Samokhvalov AV and Shield KD: Global burden of alcoholic liver diseases. J Hepatol 59: 160-168, 2013.

2. Allampati S and Mullen KD: Long-term management of alcoholic liver disease. Clin Liver Dis 20: 551-562, 2016.

3. Dugum M and McCullough A: Diagnosis and management of alcoholic liver disease. J Clin Transl Hepatol 3: 109-116, 2015.

4. Hoek JB, Cahill A and Pastorino JG: Alcohol and mitochondria: A dysfunctional relationship. Gastroenterology 122: 2049-2063, 2002

5. Ceni E, Mello T and Galli A: Pathogenesis of alcoholic liver disease: Role of oxidative metabolism. World J Gastroenterol 20 : 17756-17772, 2014.

6. Szabo G: Gut-liver axis in alcoholic liver disease Gastroenterology 148: 30-36, 2015.

7. Titz B, Elamin A, Martin F, Schneider T, Dijon S, Ivanov NV, Hoeng J and Peitsch MC: Proteomics for systems toxicology. Comput Struct Biotechnol J 11: 73-90, 2014.

8. Tzeng SC and Maier CS: Label-free proteomics assisted by affinity enrichment for elucidating the chemical reactivity of the liver mitochondrial proteome toward adduction by the lipid electrophile 4-hydroxy-2-nonenal (HNE). Front Chem 4: 2, 2016.
9. Bantscheff M, Lemeer S, Savitski MM and Kuster B: Quantitative mass spectrometry in proteomics: Critical review update from 2007 to the present. Anal Bioanal Chem 404: 939-965, 2012

10. Nan YM, Kong LB, Ren WG, Wang RQ, Du JH, Li WC, Zhao SX, Zhang YG, Wu WJ, Di HL, et al: Activation of peroxisome proliferator activated receptor alpha ameliorates ethanol mediated liver fibrosis in mice. Lipids Health Dis 12: $11,2013$.

11. Bailey SM, Andringa KK, Landar A and Darley-Usmar VM: Proteomic approaches to identify and characterize alterations to the mitochondrial proteome in alcoholic liver disease. Methods Mol Biol 447: 369-380. 2008.

12. Zhan C, Yan L, Wang L, Jiang W, Zhang Y, Xi J, Jin Y, Chen L, Shi Y, Lin Z and Wang Q: Landscape of expression profiles in esophageal carcinoma by the cancer genome atlas data. Dis Esophagus 29: 920-928, 2016.

13. Yan L, Zhan C, Wu J and Wang S: Expression profile analysis of head and neck squamous cell carcinomas using data from the cancer genome atlas. Mol Med Rep 13: 4259-4265, 2016.

14. Livak KJ and Schmittgen TD: Analysis of relative gene expression data using real-time quantitative PCR and the 2(-Delta Delta C(T)) method. Methods 25: 402-408, 2001.

15. Gene Ontology Consortium: The Gene Ontology (GO) project in 2006. Nucleic Acids Res 34: D322-D326, 2006.

16. Kanehisa M, Goto S, Kawashima S, Okuno Y and Hattori M: The KEGG resource for deciphering the genome. Nucleic Acids Res 32: D277-D280, 2004.

17. Kim DH, Lee EM, Do SH, Jeong DH and Jeong KS: Changes of the cytoplasmic proteome in response to alcoholic hepatotoxicity in rats. Int J Mol Sci 16: 18664-18682, 2015.

18. Lee SJ, Lee DE, Kang JH, Nam MJ, Park JW, Kang BS, Lee DS, Lee HS and Kwon OS: New potential biomarker proteins for alcoholic liver disease identified by a comparative proteomics approach. J Cell Biochem 118: 1189-11200, 2017.

19. Tran M, Yang Z, Liangpunsakul S and Wang L: Metabolomics analysis revealed distinct cyclic changes of metabolites altered by chronic ethanol-plus-binge andshp deficiency. Alcohol Clin Exp Res 40: 2548-2556, 2016.

20. Ki SH, Park O, Zheng M, Morales-Ibanez O, Kolls JK, Bataller R and Gao B: Interleukin-22 treatment ameliorates alcoholic liver injury in a murine model of chronic-binge ethanol feeding: Role of signal transducer and activator of transcription 3 . Hepatology 52: 1291-1300, 2010.

21. Bertola A, Mathews S, Ki SH, Wang H and Gao B: Mouse model of chronic and binge ethanol feeding (the NIAAA model). Nat Protoc 8: 627-637, 2013.

22. Choi G and Runyon BA: Alcoholic hepatitis: A clinician's guide. Clin Liver Dis 16: 371-385, 2012.

23. Mathurin P and Lucey MR: Management of alcoholic hepatitis. Drug Ther Bull 56: S39-S45, 2012.

24. Patel VJ, Thalassinos K, Slade SE, Connolly JB, Crombie A, Murrell JC and Scrivens JH: A comparison of labeling and label-free mass spectrometry-based proteomics approaches. J Proteome Res 8: 3752-3759, 2009.

25. Bantscheff M,Lemeer S, Savitski MM and Kuster B: Quantitative mass spectrometry in proteomics: Critical review update from 2007 to the present. Anal Bioanal Chem 404: 939-965, 2012.

26. Sprecher H: New advances in fatty-acid biosynthesis. Nutrition 12 (1 Suppl): S5-S7, 1996.

27. Pace CP and Stankovich MT: Oxidation-reduction properties of short-chain acyl-CoA dehydrogenase: Effects of substrate analogs. Arch Biochem Biophys 313: 261-266, 1994.

28. Ladero JM, Martinez C, Garcia-Martin E, Fernández-Arquero M, López-Alonso G, de la Concha EG, Díaz-Rubio $M$ and Agúndez JA: Polymorphisms of the glutathione S-transferases mu-1 (GSTM1) and theta-1 (GSTT1) and the risk of advanced alcoholic liver disease. Scand J Gastroenterol 40: 348-353, 2005.

29. Eaton S, Record CO and Bartlett K: Multiple biochemical effects in the pathogenesis of alcoholic fatty liver. Eur J Clin Invest 27: 719-722, 1997.

30. Cunningham CC and Bailey SM: Ethanol consumption and liver mitochondria function. Biol Signals Recept 10: 271-282, 2001.

31. Chacko BK, Srivastava A, Johnson MS, Benavides GA, Chang MJ, Ye Y, Jhala N, Murphy MP, Kalyanaraman B and Darley-Usmar VM: Mitochondria-targeted ubiquinone (MitoQ) decreases ethanol-dependent micro and macro hepatosteatosis. Hepatology 54: 153-163, 2011.

32. Sugimoto K and Takei Y: Pathogenesis of alcoholic liver disease. Hepatol Res 47: 70-79, 2016. 
33. Williams JA, Ni H, Ding Y and Ding W: Parkin regulates mitophagy and mitochondrial function to protect against alcohol-induced liver injury and steatosis in mice. Am J Physiol Gastrointest Liver Physiol 309: G324-G340, 2015.

34. Lieber CS and DeCarli LM: Ethanol oxidation by hepatic microsomes: Adaptive increase after ethanol feeding. Science 162: 917-918, 1968.

35. Lieber CS and DeCarli LM: Hepatic microsomal ethanol-oxidizing system. In vitro characteristics and adaptive properties in vivo. J Biol Chem 245: 2505-2512, 1970.

36. Robin M, Sauvage I, Grandperret T, Descatoire V, Pessayre D and Fromenty B: Ethanol increases mitochondrial cytochrome P450 2E1 in mouse liver and rat hepatocytes. Febs Lett 579: 6895-6902, 2005.

37. Cieślak A, Kelly I, Trottier J, Verreault M, Wunsch E, Milkiewicz P, Poirier G, Droit A and Barbier O: Selective and sensitive quantification of the cytochrome $\mathrm{P} 450$ 3A4 protein in human liver homogenates through multiple reaction monitoring mass spectrometry. Proteomics 16: 2827-2837, 2016.

38. Bradford BU, Kono H, Isayama F, Kosyk O, Wheeler MD Akiyama TE, Bleye L, Krausz KW, Gonzalez FJ, Koop DR and Rusyn I: Cytochrome P450 CYP2E1, but not nicotinamide adenine dinucleotide phosphate oxidase, is required for ethanol-induced oxidative DNA damage in rodent liver. Hepatology 41: 336-344, 2005.

39. Lieber CS: The discovery of the microsomal ethanol oxidizing system and its physiologic and pathologic role. Drug Metab Rev 36: 511-529, 2004.
40. Nieto N: Stimulation and proliferation of primary rat hepatic stellate cells by cytochrome P450 2E1-derived reactive oxygen species. Hepatology 35: 62-73, 2002.

41. Bridle K, Cheung TK, Murphy T, Walters M, Anderson G, Crawford DG and Fletcher LM: Hepcidin is down-regulated in alcoholic liver injury: Implications for the pathogenesis of alcoholic liver disease. Alcohol Clin Exp Res 30: 106-112, 2006.

42. Suzuki Y, Saito H, Suzuki M, Hosoki Y, Sakurai S, Fujimoto Y and Kohgo Y: Up-regulation of transferrin receptor expression in hepatocytes by habitual alcohol drinking is implicated in hepatic iron overload in alcoholic liver disease. Alcohol Clin Exp Res 26: 26S-31S, 2002.

43. Kohgo Y, Ohtake T, Ikuta K, Suzuki Y, Torimoto Y and Kato J: Dysregulation of systemic iron metabolism in alcoholic liver diseases. J Gastroenterol Hepatol 23 (Suppl 1): S78-S81, 2008.

44. Bai T, Yang Y, Yao YL, Sun P, Lian LH, Wu YL and Nan JX: Betulin alleviated ethanol-induced alcoholic liver injury via SIRT1/AMPK signaling pathway. Pharmacol Res 105: 1-12, 2016.

45. Angiuoli SV, Matalka M, Gussman A, Galens K, Vangala M, Riley DR, Arze C, White JR, White O and Fricke WF: CloVR: A virtual machine for automated and portable sequence analysis from the desktop using cloud computing. BMC Bioinformatics 12: 356, 2011.

(i) (5) $\ominus$ This work is licensed under a Creative Commons Attribution-NonCommercial-NoDerivatives 4.0 International (CC BY-NC-ND 4.0) License. 